\title{
Risk Factors for Shoulder Stiffness: Current Concepts
}

\author{
Davide Cucchi ${ }^{1,2}$ Antongiulio Marmotti ${ }^{3}$ Silvana De Giorgi ${ }^{4}$ Alberto Costa ${ }^{5}$ Rocco D'Apolito ${ }^{6}$ \\ Marco Conca ${ }^{7}$ Alessandro Russo ${ }^{8}$ Maristella F. Saccomanno ${ }^{9}$ Laura de Girolamo ${ }^{10}$ SIGASCOT Research $^{2}$ \\ Committee
}

${ }^{1}$ Dipartimento di Scienze Biomediche per la Salute, Università degli Studi di Milano, Milan, Italy

2 Department of Orthopaedics and Trauma Surgery, University of Bonn, Bonn, Germany

${ }^{3}$ Department of Orthopaedics and Traumatology, University of Torino, Turin, Italy

${ }^{4}$ Department of Basic Medical Sciences, Neurosciences and Sensory Organs, University of Bari, Bari, Italy

${ }^{5}$ Departemnt of Orthopaedics and Traumatology, Orlandi Hospital, Bussolengo, Verona, Italy

${ }^{6}$ Department of Orthopaedics, S. Giovanni Calibita Fatebenefratelli Hospital, Rome, Italy

${ }^{7}$ UO Ortopedia Clinica San Carlo, Paderno Dugnano, Milan, Italy

8 Laboratorio di Biomeccanica e Innovazione Tecnologica-Clinica II, Istituto Ortopedico Rizzoli, Bologna, Italy

${ }^{9}$ Department of Orthopaedics, Catholic University, “A. Gemelli” University Hospital, Rome, Italy

10 Laboratorio di Biotecnologie applicate all'Ortopedia, IRCCS Istituto Ortopedico Galeazzi, Milan, Italy
Address for correspondence Davide Cucchi, MD, Department of Orthopaedics and Trauma Surgery, University of Bonn, Sigmund-Freud-Straße 25, D-53127 Bonn, Germany (e-mail: d.cucchi@gmail.com).
Abstract
Keywords
- shoulder stiffness
- frozen shoulder
- adhesive capsulitis
- shoulder arthroscopy
- inflammation

Shoulder stiffness is a condition of painful restriction of the glenohumeral range of motion. Numerous risk factors for primary and postoperative shoulder stiffness have been described. This article summarizes the known aspects of the pathophysiology of shoulder stiffness, with special attention to elements of molecular biology and genetics, which could influence the risk of developing shoulder stiffness. Furthermore, the role of hormonal and metabolic factors, medical disorders, drugs, and of other published risk factors for primary and postoperative shoulder stiffness is reviewed and discussed. Finally, aspects related to shoulder surgery and postoperative rehabilitation protocols, which could influence the development of postoperative stiffness are presented.

\section{Introduction}

Shoulder stiffness (SS) is a condition of restricted glenohumeral range of motion (ROM), which can arise spontaneously (primary or idiopathic SS, also known as "frozen shoulder") or as consequence of a known cause, including surgical procedures on the shoulder (secondary and postoperative SS). Numerous risk factors have been described, both for primary and postoperative SS. ${ }^{1-4}$ The goal of this article is to describe the role of clinical conditions and intraoperative variables on the development of SS and clarify the relations between the most frequently recognized risk factors and their biological background.

\section{Molecular Biology and Genetics of Shoulder Stiffness}

Stimulation of the glenohumeral synovium, with early biochemical signs of inflammation and ischemia, is the beginning of a biological cascade resulting in fibrosis, the hallmark of glenohumeral capsule contracture, which leads to the clinical picture of SS; since generally no adhesions between layers are present, the use of the term "adhesive capsulitis" has been discouraged, as not reflecting the pathological process of this disease. ${ }^{1}$ published online December 11, 2017
DOI https://doi.org/ 10.1055/s-0037-1608951. ISSN 2282-4324.
Copyright (c) 2017 Georg Thieme Verlag License terms KG Stuttgart - New York 
Synovial cells and capsular fibroblasts are the initial target of an inflammatory-fibrotic cascade: ${ }^{5}$ a cytokine-mediated fibroblastic hyperplasia of the synovium is generated by the contemporary overexpression of transforming growth factor- $\beta$ (TGF- $\beta$ ) and its receptor, tumor necrosis factor- $\alpha$ (TNF- $\alpha$ ), platelet-derived growth factor and its receptor, hepatocyte growth factor, interleukin (IL) 1, and 6., ${ }^{6,7}$ This leads to an initial, transient hyperplasic and hypervascular environment, which contains principally synovial cells, with only occasional T-cells.

This first phase is followed by alterations of the connective tissue in the glenohumeral capsule: loss of order and twisting of collagen fibrils (resulting in up to a fourfold increase in diameter) have been observed and linked to two important features of SS: phenotypic shift of fibroblasts and imbalance in matrix metalloproteinases (MMP) homeostasis. ${ }^{7}$

Myofibroblasts are contractile cells, which play a known role in liver, lung, and kidney fibrosis and other fibrotic disorders as Dupuytren's disease. ${ }^{1}$ Their cytocontractile proteins, as vimentin, have been found also in the anterior capsular structures in patients with primary SS. The phenotypic shift from fibroblasts to myofibroblasts and their proliferation between nodular bands of collagen is therefore a key to understanding the pathology of SS. ${ }^{1}$ Phenotypic shift of fibroblasts may lead also to chondrogenic processes, with increased expression of aggrecan, as observed in the capsule of idiopathic SS. ${ }^{8}$

Imbalance in the MMP/tissue inhibitors of metalloproteinases (TIMP) ratio (the ratio of MMP-1 and MMP-2 levels against TIMP-1 and TIMP-2 levels) is another relevant factor in development of capsular fibrosis. In SS, the increased presence of TGF- $\beta$ enhances the production of TIMP- 1 and TIMP-2, whereas the production of some MMPs as MMP-1, MMP-2, and MMP-14 is negatively influenced by this cytokine. ${ }^{9}$ In opposition to other MMPs, MMP-3 (stromelysin- 1 ) level is increased, probably following IL- $1 \beta$ expression. However, as observed for liver fibrosis, MMP-3 is involved in early matrix degradation and may stimulate the conversion of fibroblasts to myofibroblasts, influencing negatively the progression of the disease. ${ }^{10}$

Increased TGF- $\beta$ stimulation is also responsible for the enhanced expression of fibronectin and tenascin, two proteins linked to fibrotic diseases. Tenascin has a role in cell adhesion, fibroblast migration, and processes related to tissue remodeling, while fibronectin is essential for collagen fibril assembly, extracellular matrix (ECM) deposition, and fibroblast activation; both seem to be involved in the fibrotic process affecting the glenohumeral capsule. ${ }^{11}$

Lastly, an increase in substance P has been reported in SS: this may represent an early element of the fibrotic cascade, since substance $P$ upregulates TGF- $\beta$ and promotes neo-angiogenesis, fibroblasts proliferation, and contraction, without directly altering ECM synthesis. ${ }^{12}$ A myofibroblast-mast cellneuropeptide axis has been described, in which the interaction of substance $P$ with the resident mast cells in the cellular microenvironment is involved in fibrosis progression. ${ }^{13}$

The fibrotic evolution of SS is therefore the final step of a biological cascade starting with cytokine hyperproduction, which results in excessive deposition of collagen type I, III, and other components of the ECM, along with inhibition of matrix degradation and phenotypic shift of resident fibroblasts toward myofibroblasts.

This inflammatory-fibrotic cascade (-Fig. $\mathbf{1}$ ), in which multiple biological elements play different roles, may be triggered by single elements, and hence considered direct risk factors for fibrotic diseases: gene polymorphisms of IL-6 and MMP-3 have been associated to increased susceptibility to SS, and other genetic polymorphism in MMPs and TGF- $\beta 1$ have been linked to different fibrotic diseases. ${ }^{14,15}$ Furthermore, in some clinical conditions with increased risk for development of SS, as hypothyroidism and diabetes, TGF- $\beta$ and TNF- $\alpha$ are hyperexpressed: ${ }^{16}$ diabetes was related to increased TGF- $\beta 1$ and TNF- $\alpha$ serum concentrations ${ }^{17,18}$ and subclinical hypothyroidism was also associated with higher serum levels of TGF- $\beta 1$ and markers of endothelial dysfunction ${ }^{19}$ and, in experimental models, with high TNF- $\alpha$ serum concentrations. ${ }^{20}$ Surprisingly, even depressive disorders, often detected as coexistent personality traits in patients with clinical pictures of SS, are related to high levels of serum TGF- $\beta$ and TNF- $\alpha .{ }^{21-23}$ These evidences seem to suggest the possibility of an underlying aspecific proinflammatory condition, characterized by increased expression of TNF- $\alpha$ and TGF- $\beta$, which may represent itself a predisposing risk factor for the development of SS. TNF- $\alpha /$ TGF- $\beta$ hyperexpression could even represent a "phenotypic pattern" underlying to multiple diseases: ${ }^{24}$ in the orthopedic field, primary SS, arthrofibrosis, and bone pathologies linked to ischemic endothelial dysfunction (as primary bone marrow edema syndrome) all show deep relations to inflammatory processes; further biological and clinical studies may allow for better elucidation of these specific concepts and confirmation of this intriguing hypothesis (-Fig. 1).

Genetics also play a role in the development of SS. As many soft tissue diseases, SS is a multifactorial process, in which genetics and environment interact to generate the final phenotypical appearance of the disease. The relative genetic and environmental contribution to idiopathic SS was first explored by Hakim et $\mathrm{al}^{25}$ by means of the classic twin model: an overall prevalence of $11.6 \%$ in 1,828 unselected female twin pairs and a heritability of $42 \%$ for idiopathic SS was identified, demonstrating that genetic factors play a role in its pathogenesis; the authors could not find evidences suggesting the influence of behavioral factors.

For what concerns familial clustering, a positive family history of idiopathic SS in a first-degree relative was reported in larger studies ${ }^{2,25,26}$ and in some case reports studying the relation between two first-degree relatives: bilateral SS in two brothers at the same time, ${ }^{27}$ in two identical twins ${ }^{28}$ and in father and daughter. ${ }^{29}$ Racial predilection is another factor generally investigated in association with genetic predisposition. A higher percentage of patients with primary SS was observed in white (76\%) rather than black subjects $(24 \%)^{30}$ and in individuals born in or having parents or grandparents born in the British Isles. ${ }^{25}$

A recent systematic review explored the association of idiopathic SS with family history and race, both of which revealed a genetic predisposition to this condition. In particular, familiarity was investigated by collecting twin and familial 


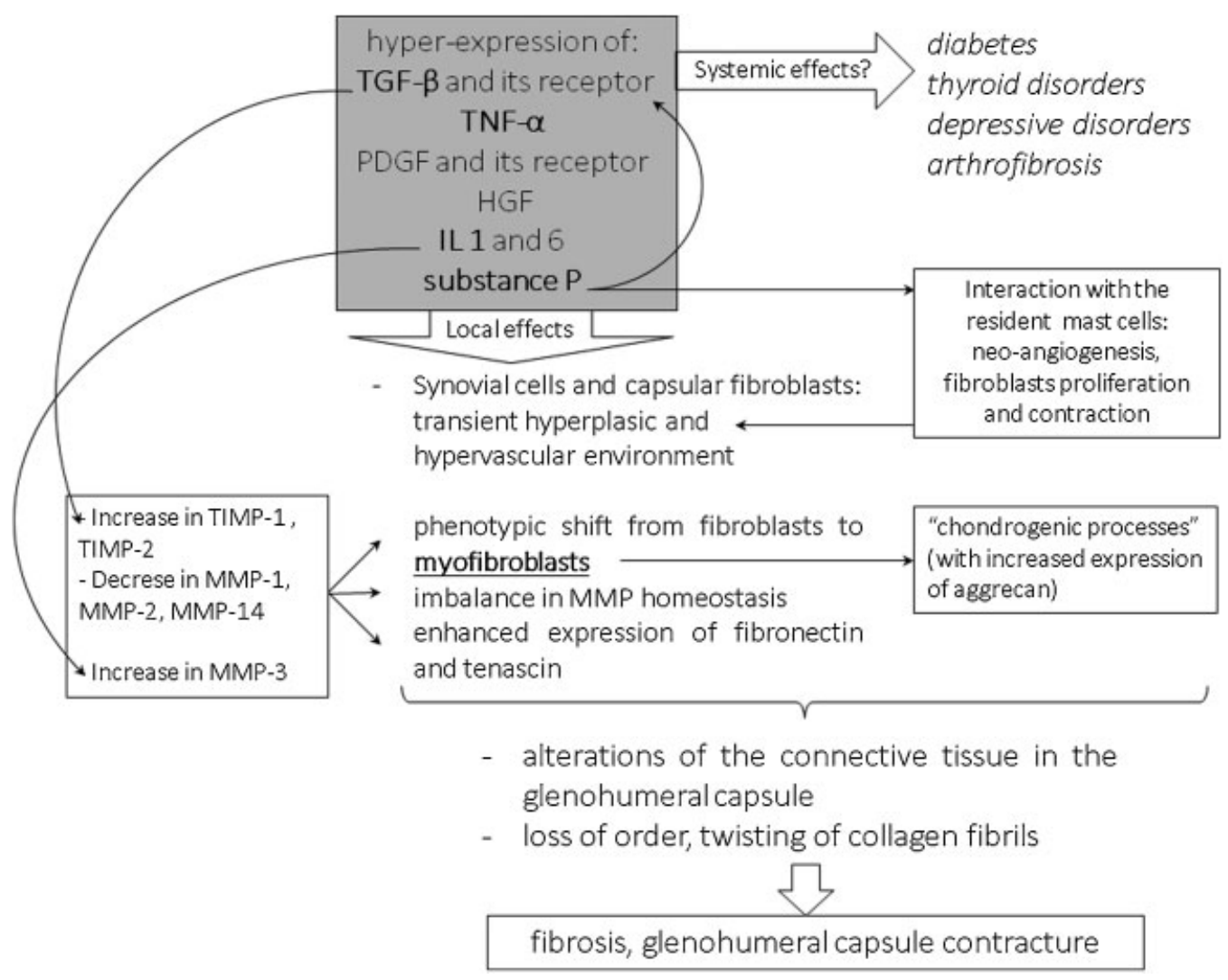

Fig. 1 Key elements of the inflammatory-fibrotic cascade underlying shoulder stiffness (SS), which may allow to hypothesize an underlying metabolic/pro-inflammatory systemic condition, characterized by TNF- $\alpha$ /TGF- $\beta$ hyperexpression. Abbreviations: HGF: hepatocyte growth factor; IL: interleukin; MMP: matrix metalloproteinases; PDGF: platelet-derived growth factor; TGF- $\beta$ : transforming growth factor- $\beta$; TIMP, tissue inhibitors of metalloproteinases; TNF- $\alpha$ : tumor necrosis factor- $\alpha$.

clustering studies. ${ }^{31}$ In the same study, the authors also performed a meta-analysis of immunological researches, which investigated the role of human leukocyte antigen (HLA)-B27, a protein involved in triggering the inflammatory response, as a risk factor for SS. Although unbiased genetic approaches were lacking and insufficient to definitively confirm this hypothesis, a significantly higher rate of HLA-B27 positivity in patients with idiopathic SS as compared with controls was reported, suggesting a genetic link with this condition. ${ }^{31}$

Being SS characterized by pain, inflammation, and fibrosis, it is also reasonable to expect genes involved in altered collagen structure and metabolism, nerve physiology, or inflammatory cytokines-mediated pathways to be altered in patients affected by SS: until now, only gene polymorphisms of IL-6 and MMP-3 have been associated to susceptibility of postoperative SS. ${ }^{14}$ Large epidemiological studies along with genome-wide association studies are needed to address definitively the molecular genetics of SS.

\section{Hormonal and Metabolic Factors, Medical Conditions, and Drugs}

Numerous clinical conditions have been related to the development of SS; in particular, the association between endocrine disorders and SS has been extensively described.

Patients with established diabetes mellitus (DM) have a greater likelihood of developing both primary and postsurgical SS than the healthy population. ${ }^{1,16,26,32}$ A recent meta-analy- sis demonstrated an overall mean prevalence of idiopathic SS in patients affected by DM of $13.4 \%$ and a mean prevalence of DM in a population with SS of $30 \%{ }^{33}$ This suggests that patients with SS should be investigated to exclude DM, since a diagnosis of SS may be the first warning sign of a diabetic condition. Postoperative SS has been reported in diabetic patients ${ }^{16,34}$ with possible explanations being the synovial neovascularisation ${ }^{35}$ and altered expression of inflammatory cytokines as TGF- $\beta$ and TNF- $\alpha{ }^{17,18,36}$

The connection between thyroid dysfunction and SS is well known: hyper- and hypothyroidism were both reported in association with primary and postoperative SS: ${ }^{37-39}$ idiopathic SS was found to be present in $17.4 \%$ of patients with subclinical hyperthyroidism, 7.1\% with hyperthyroidism, and 13\% with hypothyroidism. ${ }^{40}$ In a longitudinal population-based study, hyperthyroidism was indicated as a significant risk factor for idiopathic $\mathrm{SS},{ }^{39}$ while in a case-control study hypothyroidism was found to be associated with idiopathic SS. ${ }^{26}$ Finally, subclinical hypothyroidism was indicated as a risk factor for the development of severe postoperative SS. ${ }^{16}$ The presence of full-blown as well as subclinical thyroid disease could hence account for otherwise inexplicable SS following arthroscopic procedures; appropriate diagnostic and prophylactic measures prior to shoulder surgery are therefore advised.

No studies regarding calcitonine levels and SS have been published yet; however, the use of calcitonine, both subcutaneous and as intranasal spray, was reported as effective in 
reducing pain and improving function of stiff shoulders. ${ }^{41}$ A single report on the association between adrenocorticotropic hormone $(\mathrm{ACTH})$ deficiency and SS has been published. ${ }^{42}$

Women are more frequently affected by SS than men, 2,43 suggesting that sexual hormones contribute to SS etiology; however, no published evidence has yet related female sexual hormones with a SS. Whether the lower level of testosterone in women of reproductive age could influence the epidemiology of SS is also unknown; the fact that testosterone may affect the TGF- $\beta$ signaling pathway suggests a possible field for further investigation. ${ }^{44}$

Neurologic conditions were also associated with primary SS: surgical treatment for subarachnoid hemorrhage was associated to a $25 \%$ incidence of $\mathrm{SS}^{45}$ and a case-control study reported a significantly higher incidence of SS (12.7\% versus $1.7 \%$ ) in patients affected by Parkinson's disease. ${ }^{46}$ Injuries to the cervical spine or brachial plexus were also mentioned among the possible etiologies for secondary SS. ${ }^{1}$

Psychological factors may also play a role in the development of SS, although the causative relation between these and SS has not yet been clearly defined. ${ }^{22,47}$

Dupuytren's disease was found in 52\% of the patients diagnosed with primary SS, indicating an 8.27 times higher frequency of this disease in patients with SS than in the general population. ${ }^{48}$ A possible common origin of this two diseases was suggested by Hutchinson et al, who observed an unusually high incidence of SS and Dupuytren's disease after treatment with a now withdrawn synthetic MMP inhibitor (Marimastat). ${ }^{49}$

Different malignant diseases are associated to musculoskeletal manifestations, with SS being reported with a $15 \%$ incidence in an oncologic population; this value raised to $17.8 \%$ when considering patients affected by solid malignancies only. ${ }^{50}$ Development of SS was also reported after invasive procedures for breast cancer treatments or cardiac catheterization and open procedures on the shoulder. ${ }^{1}$

The role of lipid metabolism in SS has been scarcely investigated: in a prospective study, the fasting serum triglyceride and cholesterol levels were significantly elevated in patients diagnosed with idiopathic SS; more recently, hypercholesterolemia and inflammatory lipoproteinemias were demonstrated to have a significant association with primary SS. ${ }^{51,52}$

Several reports show that SS can develop after treatment with specific medications. Patients treated with protease inhibitors during highly active antiretroviral therapy (HAART) for HIV infection may develop SS. One or both shoulders can be affected and the disease may occur from some weeks to several months after the beginning of the therapy. It has been hypothesized that the pathologic mechanism could involve an enhancement of TGF- $\beta$ pathway. The local trigger could be a precipitation of the drug in the joint, leading to fibrosis as result. $^{53,54}$ The majority of reports dealt with indinavir, but there is also a report of SS in a patient treated with nelfinavir. ${ }^{55}$

MMPs inhibitors were associated with the development of primary SS: six patients suffering from bilateral SS after 4 months of treatment with Marimastat for advanced gastric carcinoma were reported in a series of 12 patients treated for more than 1 month. The postulated mechanism in these cases involved the decrease in MMP/TIMP ratio, which caused increased synthesis and deposition of collagen and connective tissue. Interestingly, the acute symptoms of SS improved when treatment with Marimastat was temporarily discontinued. $^{49}$ The same mechanism was advocated for bilateral SS observed in a patient after 12 cycles of FOLFOX (folinic acid, 5-fluorouracil, and oxaliplatin); indeed the last two drugs inhibit MMP production. ${ }^{56}$

Historical reports of connective tissue disorders related to barbiturates were confirmed in a prospective survey of patients treated for epilepsy in the 1980s. Two patients treated with primidone developed primary SS, one patient treated with phenobarbital suffered from shoulder pain whereas seven patients developed connective tissue disorders in different anatomical regions out of 622 subjects involved in the study. The onset of SS with such medications is highly variable, ranging from some months after starting the therapy to many years later. The authors mentioned as possible trigger the decreased activity of prostaglandin E caused by antiepileptic drugs which could influence fibroblast activation. ${ }^{57}$

Isoniazid and ethionamide, two antituberculous drugs, were associated with the development of primary SS: in these cases, the onset was rapid, usually occurring within a month after therapy is started. The theorized pathologic mechanisms involved direct effects on collagen metabolism and inhibition of serotonin degradation, which, in turn, is involved in tissue fibrosis. ${ }^{58}$ Pefloxacin (a fluoroquinolone) was ascribed as possible cause of SS in two patients, a man treated for urinary tract infection and a diabetic woman treated for meningitis after undergoing lumbar discectomy. ${ }^{59}$

Postvaccination SS syndromes were reported: in all cases, SS followed an intramuscular deltoid injection of a vaccine (influenza, hepatitis-A, tetanus, pneumococcal). The course was characterized by the onset of progressively increasing shoulder pain in the first few days after the injection; fullblown SS could arise in the following months. It was suggested the trigger to be the accidental infiltration of the subacromial bursa. ${ }^{60}$

Tobacco smoke is known to affect ECM homeostasis, altering the levels of MMPs and their inhibitors and is frequently reported as a risk factor for SS. ${ }^{1}$ Although negative effects of smoke on shoulder ROM and tissue quality were described, no direct associations between development of SS and smoking habits could be identified in the published literature. ${ }^{61-63}$

\section{Surgical Factors and Rehabilitation}

Arthroscopic rotator cuff ( $\mathrm{RC}$ ) repair is considered the gold standard in surgical treatment of RC tears and has proven to be effective, safe, and with a durable clinical success rate. ${ }^{64,65}$ However, postoperative stiffness following RC repair has been reported to occur with an incidence ranging between 1.5 and $32.7 \%{ }^{66,67}$ In the largest consecutive published series, the overall rate of postoperative stiffness (defined as "patients' dissatisfaction with their ROM") was $4.9 \%{ }^{3}$ Postoperative SS typically presents after an initially successful rehabilitation period, with increasing shoulder pain and with gradual loss of both active and passive ROM; although most cases resolve within 6 months, established SS may develop, with devastating consequences. ${ }^{68}$ 
The exact etiology of postoperative SS has not been established yet: capsular contractures and postsurgical adhesion to the surrounding soft tissues are considered to be responsible for development of postoperative SS. ${ }^{1}$ In addition to the hormonal factors mentioned above, the risk of developing postoperative SS was also associated with age less than 50 years, ${ }^{3,4}$ presence of gastroesophageal disorders, ${ }^{69}$ calcific tendonitis or idiopathic SS of the index shoulder, ${ }^{3}$ higher grade of fatty infiltration, ${ }^{70}$ and Workers' Compensation. ${ }^{3,4}$ Lowgrade infection by Propionibacterium acnes has also been proposed as a possible cause for postoperative SS. ${ }^{71,72}$

Some intrinsic features of a RC tear were related to higher risk of postoperative SS: posterosuperior tears showed a statistically significantly higher prevalence of postoperative stiffness, as did posttraumatic tears. ${ }^{66}$ For what concerns lesions' type and dimensions, Huberty et al, observed that patients with single-tendon tears, smaller tears, and partial articular-sided tendon avulsion lesions were more likely to develop postoperative SS than those with full-thickness tears, larger in size, and/or involving more tendons. ${ }^{3}$ On the contrary, others indicated larger tear size as associated to an increased risk for postoperative SS. ${ }^{66,70}$ Patients undergoing concomitant labral repair ${ }^{3}$ or open or miniopen repair $^{70}$ also showed a higher incidence of postoperative stiffness, whereas patients with concomitant coracoplasty were less likely to develop postoperative $\mathrm{SS}^{3}{ }^{3}$ When investigated, the fixation technique (single- versus double-row) did not appear to influence the incidence of postoperative SS. ${ }^{73}$

Rehabilitation may also play a role in the development of postoperative SS after arthroscopic RC repair, and an ideal rehabilitation protocol that allows for tendon-to-bone healing while preventing SS has not been established yet.

Basic science studies showed that too-rapid advancing motion protocols could lead to an inflammatory response, which increases the risk of postoperative adhesions, and produces strain on the RC with potential higher risk of retear. ${ }^{74-76}$ On the contrary, early restriction of motion did not appear to cause long-term stiffness, even in patients who develop clinical stiffness in the early postoperative period. ${ }^{75}$ Therefore, the majority of the rehabilitation protocols after $\mathrm{RC}$ repair are based on an initial phase of restricted ROM and clinical observation, to improve the rate of tendon healing. ${ }^{75}$ In this phase, postoperative positioning of the arm in an abduction sling can help keeping the inferior glenohumeral joint capsule stretched out. ${ }^{1}$

Nevertheless, a role in early postoperative motion to prevent SS has been suggested: Denard et al observed that postoperative SS was reduced from $4.5 \%$ in patient who underwent 6 weeks' immobilization protocol to $1.5 \%$ in patients who started immediate recovery of passive $\mathrm{ROM} .{ }^{4}$ Scapular kinematics may also be involved in the development of postoperative SS because alterations in scapular position and motion occur in the vast majority of patients after shoulder injury or surgery. Since scapular dyskinesis may cause restriction of motion leading to SS, particular attention should be paid to include scapular rehabilitation in postoperative protocols. ${ }^{77}$

Finally, tailoring rehabilitation taking into consideration preoperative and intraoperative risk factors demonstrated to reduce the incidence of SS: patients at risk for SS enrolled in a modified rehabilitation protocol that added immediate closedchain passive forward flexion exercise to a standard protocol consisting of sling immobilization without overhead shoulder motion for 6 weeks showed, in facts, successful reduction of postoperative SS incidence. ${ }^{78}$ Therefore, a specialized shoulder physiotherapy, carefully instructed to focus on the development of postoperative SS and its immediate recognition and treatment, is recommended. $^{79}$

\section{Conclusion}

The exact etiology of SS remains debated and numerous risk factors for this pathology have been proposed. The role of some hormonal and metabolic diseases, such as diabetes, hyper-, and hypothyroidism, has been demonstrated to be relevant role in the development of SS. Numerous other potentially relevant factors have been described, which require further research to be confirmed. Recent evidences from molecular biology suggest that an underlying aspecific pro-inflammatory condition could represent a predisposing risk factor for the development of SS. Postoperative stiffness can be triggered by aspects related to shoulder surgery and postoperative rehabilitation protocols.

\section{Conflict of Interest}

Dr de Girolamo reports personal fees from Lipogems and Geistlich and grants from IGEA, outside the submitted work. All the other authors report no conflict of interest.

\section{References}

1 Itoi E, Arce G, Bain GI, et al. Shoulder stiffness: current concepts and concerns. Arthroscopy 2016;32(07):1402-1414

2 Hand G, Carr A. Natural history and genetics of frozen shoulder. A 1-20 year follow up of 273 patients. Orthop Proc 2005;87:xx

3 Huberty DP, Schoolfield JD, Brady PC, Vadala AP, Arrigoni P, Burkhart SS. Incidence and treatment of postoperative stiffness following arthroscopic rotator cuff repair. Arthroscopy 2009;25 (08):880-890

4 Denard PJ, Lädermann A, Burkhart SS. Prevention and management of stiffness after arthroscopic rotator cuff repair: systematic review and implications for rotator cuff healing. Arthroscopy 2011;27(06):842-848

5 Rodeo SA, Hannafin JA, Tom J, Warren RF, Wickiewicz TL. Immunolocalization of cytokines and their receptors in adhesive capsulitis of the shoulder. J Orthop Res 1997;15(03):427-436

6 Lho Y-M, Ha E, Cho C-H, et al. Inflammatory cytokines are overexpressed in the subacromial bursa of frozen shoulder. J Shoulder Elbow Surg 2013;22(05):666-672

7 Le HV, Lee SJ, Nazarian A, Rodriguez EK. Adhesive capsulitis of the shoulder: review of pathophysiology and current clinical treatments. Shoulder Elbow 2017;9(02):75-84

8 Hagiwara Y, Ando A, Onoda Y, et al. Coexistence of fibrotic and chondrogenic process in the capsule of idiopathic frozen shoulders. Osteoarthritis Cartilage 2012;20(03):241-249

9 Lubis AMT, Lubis VK. Matrix metalloproteinase, tissue inhibitor of metalloproteinase and transforming growth factor-beta 1 in frozen shoulder, and their changes as response to intensive stretching and supervised neglect exercise. J Orthop Sci 2013; 18(04):519-527 
10 Han Y-P. Matrix metalloproteinases, the pros and cons, in liver fibrosis. J Gastroenterol Hepatol 2006;21(Suppl 3):S88-S91

11 Cohen C, Leal MF, Belangero PS, et al. The roles of tenascin C and fibronectin 1 in adhesive capsulitis: a pilot gene expression study. Clinics (Sao Paulo) 2016;71(06):325-331

12 Fong G, Backman LJ, Alfredson H, Scott A, Danielson P. The effects of substance $P$ and acetylcholine on human tenocyte proliferation converge mechanistically via TGF- $\beta 1$. PLoS One 2017;12(03): e0174101

13 Hildebrand KA, Zhang M, Befus AD, Salo PT, Hart DA. A myofibroblast-mast cell-neuropeptide axis of fibrosis in post-traumatic joint contractures: an in vitro analysis of mechanistic components. J Orthop Res 2014;32(10):1290-1296

14 Ling Y, Peng C, Liu C, Zhang N, Yue S. Gene polymorphism of IL-6 and MMP-3 decreases passive range of motion after rotator cuff repair. Int J Clin Exp Pathol 2015;8(05):5709-5714

15 Martelossi Cebinelli GC, Paiva Trugilo K, Badaró Garcia S, Brajão de Oliveira K. TGF- $\beta 1$ functional polymorphisms: a review. Eur Cytokine Netw 2016;27(04):81-89

16 Blonna D, Fissore F, Bellato E, et al. Subclinical hypothyroidism and diabetes as risk factors for postoperative stiff shoulder. Knee Surg Sport Traumatol Arthrosc 2015;25(07):2208-2216

17 Qiao Y-C, Chen Y-L, Pan Y-H, et al. Changes of transforming growth factor beta 1 in patients with type 2 diabetes and diabetic nephropathy: a PRISMA-compliant systematic review and meta-analysis. Medicine (Baltimore) 2017;96(15):e6583

18 Chen Y-L, Qiao Y-C, Xu Y, et al. Serum TNF- $\alpha$ concentrations in type 2 diabetes mellitus patients and diabetic nephropathy patients: a systematic review and meta-analysis. Immunol Lett 2017;186:52-58

19 Arpaci D, Karakece E, Tocoglu AG, et al. Endocan, TGF-beta, and ADMA as risk factors for endothelial dysfunction and possible vascular disease in patients with subclinical hypothyroidism. Ann Clin Lab Sci 2016;46(06):601-607

20 Iams WT, Hames ML, Tsai JP, et al. Increased serum tumor necrosis factor $\alpha$ levels in patients with lenalidomide-induced hypothyroidism. Exp Hematol 2015;43(02):74-78

21 Köhler CA, Freitas TH, Maes M, et al. Peripheral cytokine and chemokine alterations in depression: a meta-analysis of 82 studies. Acta Psychiatr Scand 2017;135(05):373-387

22 Ding H, Tang Y, Xue Y, et al. A report on the prevalence of depression and anxiety in patients with frozen shoulder and their relations to disease status. Psychol Health Med 2014;19(06): 730-737

23 Davami MH, Baharlou R, Ahmadi Vasmehjani A, et al. Elevated IL-17 and TGF- $\beta$ serum levels: a positive correlation between T-helper 17 cell-related pro-inflammatory responses with major depressive disorder. Basic Clin Neurosci 2016;7(02):137-142

24 Pietrzak M. Adhesive capsulitis: an age related symptom of metabolic syndrome and chronic low-grade inflammation? Med Hypotheses 2016;88:12-17

25 Hakim AJ, Cherkas LF, Spector TD, MacGregor AJ. Genetic associations between frozen shoulder and tennis elbow: a female twin study. Rheumatology (Oxford) 2003;42(06):739-742

26 Wang K, Ho V, Hunter-Smith DJ, Beh PS, Smith KM, Weber AB. Risk factors in idiopathic adhesive capsulitis: a case control study. J Shoulder Elbow Surg 2013;22(07):e24-e29

27 Toussirot E, Lohse A, Auge B, Wendling D. Bilateral frozen shoulder at the same time in two brothers. Rev Rhum Engl Ed 1999;66(79):437

28 Hirschhorn P, Schmidt JM. Frozen shoulder in identical twins. Joint Bone Spine 2000;67(01):75-76

29 Harzy T, Benbouazza K, Amine B, Rahmouni R, Guedira N, HajjajHassouni N. Idiopathic hypoparathyroidism and adhesive capsulitis of the shoulder in two first-degree relatives. Joint Bone Spine 2004;71(03):234-236

30 Rizk TE, Pinals RS. Histocompatibility type and racial incidence in frozen shoulder. Arch Phys Med Rehabil 1984;65(01):33-34
31 Prodromidis AD, Charalambous CP. Is there a genetic predisposition to frozen shoulder?: a systematic review and meta-analysis JBJS Rev 2016;4(02): Doi: 10.2106/JBJS.RVW.0.00007

32 Juel NG, Brox JI, Brunborg C, Holte KB, Berg TJ. Very high prevalence of frozen shoulder in patients with type 1 diabetes of $\geq 45$ years' duration: the Dialong Shoulder Study. Arch Phys Med Rehabil 2017;98(08):1551-1559

33 Zreik NH, Malik RA, Charalambous CP. Adhesive capsulitis of the shoulder and diabetes: a meta-analysis of prevalence. Muscles Ligaments Tendons J 2016;6(01):26-34

34 Chen AL, Shapiro JA, Ahn AK, Zuckerman JD, Cuomo F. Rotator cuff repair in patients with type I diabetes mellitus. J Shoulder Elbow Surg 2003;12(05):416-421

35 Ryu JD, Kirpalani PA, Kim JM, Nam KH, Han CW, Han SH. Expression of vascular endothelial growth factor and angiogenesis in the diabetic frozen shoulder. J Shoulder Elbow Surg 2006;15(06): 679-685

36 Kabbabe B, Ramkumar S, Richardson M. Cytogenetic analysis of the pathology of frozen shoulder. Int J Shoulder Surg 2010;4(03):75-78

37 Vicenti G, Moretti L, De Giorgi S, et al. Thyroid and shoulder diseases: the bases of a linked channel. J Biol Regul Homeost Agents 2016;30(03):867-870

38 Schiefer M, Teixeira PFS, Fontenelle C, et al. Prevalence of hypothyroidism in patients with frozen shoulder. J Shoulder Elbow Surg 2017;26(01):49-55

39 Huang SW, Lin JW, Wang WT, Wu CW, Liou TH, Lin HW. Hyperthyroidism is a risk factor for developing adhesive capsulitis of the shoulder: a nationwide longitudinal population-based study. Sci Rep 2014;4:4183

40 Cakir M, Samanci N, Balci N, Balci MK. Musculoskeletal manifestations in patients with thyroid disease. Clin Endocrinol (Oxf) 2003;59(02):162-167

41 Brue S, Valentin A, Forssblad M, Werner S, Mikkelsen C, Cerulli G. Idiopathic adhesive capsulitis of the shoulder: a review. Knee Surg Sports Traumatol Arthrosc 2007;15(08):1048-1054

42 Choy EH, Corkill MM, Gibson T, Hicks BH. Isolated ACTH deficiency presenting with bilateral frozen shoulder. $\mathrm{Br} J$ Rheumatol 1991;30 (03):226-227

43 White D, Choi H, Peloquin C, Zhu Y, Zhang Y. Secular trend of adhesive capsulitis. Arthritis Care Res (Hoboken) 2011;63(11):1571-1575

44 Braga M, Bhasin S, Jasuja R, Pervin S, Singh R. Testosterone inhibits transforming growth factor- $\beta$ signaling during myogenic differentiation and proliferation of mouse satellite cells: potential role of follistatin in mediating testosterone action. Mol Cell Endocrinol 2012;350(01):39-52

45 Bruckner FE, Nye CJ. A prospective study of adhesive capsulitis of the shoulder ("frozen shoulder') in a high risk population. QJ Med 1981;50(198):191-204

46 Riley D, Lang AE, Blair RD, Birnbaum A, Reid B. Frozen shoulder and other shoulder disturbances in Parkinson's disease. J Neurol Neurosurg Psychiatry 1989;52(01):63-66

47 Debeer P, Franssens F, Roosen I, Dankaerts W, Claes L. Frozen shoulder and the Big Five personality traits. J Shoulder Elbow Surg 2014;23(02):221-226

48 Smith SP, Devaraj VS, Bunker TD. The association between frozen shoulder and Dupuytren's disease. J Shoulder Elbow Surg 2001;10 (02):149-151

49 Hutchinson JW, Tierney GM, Parsons SL, Davis TR. Dupuytren's disease and frozen shoulder induced by treatment with a matrix metalloproteinase inhibitor. J Bone Joint Surg Br 1998;80(05):907-908

50 Gheita TA, Ezzat Y, Sayed S, El-Mardenly G, Hammam W. Musculoskeletal manifestations in patients with malignant disease. Clin Rheumatol 2010;29(02):181-188

51 Bunker TD, Esler CN. Frozen shoulder and lipids. J Bone Joint Surg Br 1995;77(05):684-686

52 Sung C-M, Jung TS, Park HB. Are serum lipids involved in primary frozen shoulder? A case-control study. J Bone Joint Surg Am 2014; 96(21):1828-1833 
53 Peyriere H, Mauboussin JM, Rouanet I, Rouveroux P, Hillaire-Buys D, Balmes P. Frozen shoulder in HIV patients treated with indinavir: report of three cases. AIDS 1999;13(16):2305-2306

54 De Ponti A, Viganò MG, Taverna E, Sansone V. Adhesive capsulitis of the shoulder in human immunodeficiency virus-positive patients during highly active antiretroviral therapy. J Shoulder Elbow Surg 2006;15(02):188-190

55 de Witte S, Bonnet F, Bonarek M, et al. Adhesive capsulitis of the shoulder in an HIV patient treated with nelfinavir. AIDS 2002;16 (09):1307-1308

56 Patel K, Patel N, Curtis M. Bilateral simultaneous frozen shoulder: a possible adverse event of the FOLFOX chemotherapy regime? Shoulder Elbow 2012;4(03):193-195

57 Mattson RH, Cramer JA, McCutchen CB. Barbiturate-related connective tissue disorders. Arch Intern Med 1989;149(04):911-914

58 Good AE, Green RA, Zarafonetis CJ. Rheumatic symptoms during tuberculosis therapy. A manifestation of isoniazid toxicity? Ann Intern Med 1965;63(05):800-807 Accessed April 26, 2017

59 Freiss S, Lecocq J, Isner ME, Vautravers P. Frozen shoulder and fluoroquinones. Two case reports. Joint Bone Spine 2000;67(03): 245-249

60 Degreef I, Debeer P. Post-vaccination frozen shoulder syndrome. Report of 3 cases. Acta Chir Belg 2012;112(06):447-449

61 Bishop JY, Santiago-Torres JE, Rimmke N, Flanigan DC. Smoking predisposes to rotator cuff pathology and shoulder dysfunction: a systematic review. Arthroscopy 2015;31(08):1598-1605

62 Kane S, Conus S, Haltom D, Hirshorn K, Pak Y, Vigdorchik J. A shoulder health survey: correlating behaviors and comorbidities with shoulder problems. Sports Health 2010;2(02):119-134

63 DE Giorgi S, Saracino M, Castagna A. Degenerative disease in rotator cuff tears: what are the biochemical and histological changes? Joints 2014;2(01):26-28

64 Spennacchio P, Banfi G, Cucchi D, D’Ambrosi R, Cabitza P, Randelli P. Long-term outcome after arthroscopic rotator cuff treatment. Knee Surg Sports Traumatol Arthrosc 2015;23(02):523-529

65 Randelli P, Cucchi D, Ragone V, de Girolamo L, Cabitza P, Randelli M. History of rotator cuff surgery. Knee Surg Sports Traumatol Arthrosc 2015;23(02):344-362

66 Seo SS, Choi JS, An KC, Kim JH, Kim SB. The factors affecting stiffness occurring with rotator cuff tear. J Shoulder Elbow Surg 2012;21(03):304-309
67 Randelli P, Spennacchio P, Ragone V, Arrigoni P, Casella A, Cabitza P. Complications associated with arthroscopic rotator cuff repair: a literature review. Musculoskelet Surg 2012;96(01):9-16

68 Evans JP, Guyver PM, Smith CD. Frozen shoulder after simple arthroscopic shoulder procedures: what is the risk? Bone Joint J 2015;97-B(07):963-966

69 Cucchi D, Menon A, Feroldi FM, et al. Risk factors for postoperative shoulder stiffness: are there new candidates? J Biol Regul Homeost Agents 2016;30(04, Suppl 1):123-129

70 Chung SW, Huong CB, Kim SH, Oh JH. Shoulder stiffness after rotator cuff repair: risk factors and influence on outcome. Arthroscopy 2013;29(02):290-300

71 Khan U, Torrance E, Townsend R, Davies S, Mackenzie T, Funk L. Low-grade infections in nonarthroplasty shoulder surgery. J Shoulder Elbow Surg 2017;26(09):1553-1561

72 Horneff JG III, Hsu JE, Voleti PB, O'Donnell J, Huffman GR. Propionibacterium acnes infection in shoulder arthroscopy patients with postoperative pain. J Shoulder Elbow Surg 2015;24(06):838-843

73 Saridakis P, Jones G. Outcomes of single-row and double-row arthroscopic rotator cuff repair: a systematic review. J Bone Joint Surg Am 2010;92(03):732-742

74 Peltz CD, Dourte LM, Kuntz AF, et al. The effect of postoperative passive motion on rotator cuff healing in a rat model. J Bone Joint Surg Am 2009;91(10):2421-2429

75 Parsons BO, Gruson KI, Chen DD, Harrison AK, Gladstone J, Flatow EL. Does slower rehabilitation after arthroscopic rotator cuff repair lead to long-term stiffness? J Shoulder Elbow Surg 2010; 19(07):1034-1039

76 Sonnabend DH, Howlett CR, Young AA. Histological evaluation of repair of the rotator cuff in a primate model. J Bone Joint Surg Br 2010;92(04):586-594

77 Kibler WB, McMullen J. Scapular dyskinesis and its relation to shoulder pain. J Am Acad Orthop Surg 2003;11(02):142-151

78 Koo SS, Parsley BK, Burkhart SS, Schoolfield JD. Reduction of postoperative stiffness after arthroscopic rotator cuff repair: results of a customized physical therapy regimen based on risk factors for stiffness. Arthroscopy 2011;27(02):155-160

79 Koorevaar RCT, Van't Riet E, Ipskamp M, Bulstra SK. Incidence and prognostic factors for postoperative frozen shoulder after shoulder surgery: a prospective cohort study. Arch Orthop Trauma Surg 2017;137(03):293-301 